\title{
Dinâmica e biologia de uma população de capivaras em ambiente antrópico, Curitiba-PR
}

\author{
Dynamics and biology of a population of capybara in anthropic \\ environment, Curitiba-PR \\ Ariádina Maria Reis de Almeida ${ }^{1}$, Daniela Biondi², Emygdio Leite de Araújo Monteiro Filho ${ }^{3}$ \\ ${ }^{1,2,3}$ Universidade Federal do Paraná, Curitiba - PR - Brasil
}

\begin{abstract}
Resumo
Esta pesquisa teve como objetivo acompanhar a evolução de uma população de capivaras presente em uma área verde urbana da capital do Estado do Paraná. Através de observações e da contagem direta dos animais, foram obtidos dados referentes ao tamanho da população, proporção de machos, de fêmeas e de animais solitários, além de tamanho e composição de grupos. Foram analisadas a densidade bruta dos quatro anos amostrados (2008 a 2011) e o número de capivaras e a variação sazonal da população durante os dois últimos anos de estudo (2010 e 2011). Os resultados obtidos indicaram que a redução da população ao longo do tempo pode estar relacionada ao controle de crescimento populacional, sendo verificado principalmente pela diminuição do número de filhotes e pela dispersão de animais para áreas vizinhas. Também se pode observar que a densidade na área de estudo corresponde a de ambientes sob influência humana, que a proporção de machos solitários é menor que em ambiente natural e que a proporção entre machos e fêmeas é semelhante a de ambientes mais preservados. Além disso, foi verificado maior número de capivaras no período mais seco de Curitiba, no outono. Conclui-se que a estrutura e composição da população de capivaras no Parque Municipal do Tingui são muito semelhantes a dos animais que vivem em áreas mais preservadas.
\end{abstract}

Palavras-chave: Densidade, Hydrochoerus hydrochaeris, Parque Municipal

\begin{abstract}
This research aimed to follow the evolution of a capybara population present in an urban green area of the capital of the State of Parana. By direct counting of animals and observing behavior, data were obtained for the population size, proportion of males, female sand solitary animals, and size and composition of groups. We analyzed the gross density of the four sampled years (2008 to 2011) and the number of capybaras and seasonal variation in population during the last two years of study (2010 and 2011). The results indicated that reducing the population over time may be related to the control of population growth being checked primarily by decreasing the number of pups and the dispersal of animals to neighboring areas. It can also be seen that the density in the study area corresponds to human environment sunder the influence that the proportion of solitary male is lower than in the natural environment and that the ratio between males and females is similar to most environments preserved. Furthermore, it was found more capybaras during the drier period of Curitiba, in the fall. We conclude that the structure and composition of the population of capybaras in Municipal Park Tingui are very similar to the animals that live in the most preserved areas.
\end{abstract}




\section{Introdução}

A capivara (Hydrochoerus hydrocheris Linaeus, 1766), um roedor de grande porte é um herbívoro da fauna brasileira que apresenta hábito semiaquático e está associado a ambientes úmidos e corpos d'água (ALHO et al., 1987b; EMMONS \& FERR, 1997). É uma espécie social que vive em grupos e apresenta um repertório comportamental complexo fundamental para a manutenção da espécie (HERRERA \& MACDONALD, 1987; ALHO et al., 1987a; SCHALLER \& CRAWSHAW, 1981).

O tamanho da população varia de acordo com as condições do ambiente e com a pressão de predação (ALDANA-DOMÍNGUEZ et al., 2007). A reprodução ocorre o ano todo, sofrendo variações em virtude de diferenças locais (DEUTSCH \& PUGLIA, 1990). Em condições favoráveis, a capivara pode ter duas crias no mesmo ano e o número de filhotes por ninhada pode variar de um a oito. Atingem a maturidade sexual entre 15 e 24 meses de idade (OLIVEIRA \& BONVICINO, 2011) e a longevidade é de 12 anos em cativeiro (DEUTSCH \& PUGLIA, 1990). Todas estas características podem variar de acordo com a estação do ano e a qualidade do ambiente (MONES \& OJASTI, 1986). De hábito diurno, a capivara se alimenta nas primeiras e últimas horas do dia e descansa nos períodos mais quentes. O descanso ocorre com mais frequência na margem da água e o forrageio em áreas mais distantes (MACDONALD, 1981; OLIVEIRA et al., 2005). Em regiões mais urbanas, este padrão de atividades pode variar de acordo com a presença humana e a pressão de caça (PEREIRA \& ESTON, 2007; VARGAS et al., 2007).

Devido a sua alta adaptabilidade, estes animais podem sobreviver também em ambientes alterados, desde que estes ofereçam abrigo, alimento e proteção, que para as capivaras significa uma fonte de água (rio, lago ou barragem), um ambiente florestal e uma cobertura vegetal para forragear (FERRAZ et al., 2009). Parecem se beneficiar da transformação das florestas em agricultura e pastos, onde os recursos alimentares são mais abundantes e estimulam o crescimento da população (FERRAZ et al., 2003; FERRAZ et al., 2007). A conectividade destes ambientes alterados permite a invasão de áreas onde antes não existiam esses animais, modificando o padrão de distribuição e a dinâmica da população (KRAUER, 2009).

Na capital do Estado do Paraná, as capivaras estão amplamente distribuídas nas inúmeras áreas verdes da região (ALMEIDA et al., 2013). Estas áreas estão geralmente localizadas ao longo de rios que drenam a cidade de Curitiba e apresentam uma extensa área de lago, característica fundamental para a ocorrência de
Hydrochoerus hydrocheris em um ambiente.

Entre as pesquisas realizadas sobre a espécie no estado do Paraná, destacam-se a análise dos comportamentos (ALMEIDA et al., 2012) de uma população de capivara presente em um parque público de Curitiba-PR, Parque Municipal Tingui, além do estudo da paisagem (ALMEIDA \& BINDI, 2011) e do microclima (ALMEIDA et al., 2013) deste parque. Para contribuir com mais informações sobre a espécie, principalmente em áreas urbanas, esta pesquisa teve como objetivo continuar estudando os animais que ocorre no Parque Municipal Tingui, dando ênfase à dinâmica e a biologia da população. Os resultados obtidos poderão contribuir não apenas para a conservação da fauna silvestre, mas também serão fontes relevantes de informação para planos de manejo da espécie em ambientes antrópicos.

No estado do Paraná, estudos recentes realizados em Curitiba sobre a paisagem (ALMEIDA \& BINDI, 2011) e o microclima (ALMEIDA et al., 2013) de um parque público e sobre os comportamentos (ALMEIDA et al., 2012) de uma população de capivaras que ocorre neste parque, dão início ao entendimento da espécie em grandes centros urbanos. Para contribuir com mais informações sobre a espécie, principalmente em áreas urbanas, esta pesquisa teve como objetivo continuar estudando os animais abordados pelos pesquisadores citados anteriormente, dando ênfase à dinâmica e a biologia da população. Os resultados obtidos poderão contribuir não apenas para a conservação da fauna silvestre, mas também serão fontes relevantes de informação para planos de manejo da espécie em ambientes antrópicos.

\section{Material e Métodos}

Esta pesquisa foi desenvolvida no Parque Municipal Tingui, uma área verde urbana localizada ao norte do Município de Curitiba, Paraná (2523'30"S e $\left.49^{\circ} 18^{\prime} 22^{\prime \prime} \mathrm{W}\right)$. Compreende uma área de 38 hectares, formada por uma paisagem heterogênea composta pelo rio Barigui, vários lagos, extensa área de gramado e pequenos remanescentes de Floresta Ombrófila Mista Aluvial. É um dos inúmeros parques públicos da cidade cuja visitação diária média é de mais de 1.600 pessoas (ECOTÉCNICA, 2009; IPPUC, 2011) (Figura 1).

Os dados de população foram obtidos em três etapas: (a) abril e março de 2008, (b) novembro de 2009 e (c) janeiro de 2010 a dezembro de 2011. As capivaras foram contadas e observadas no dia 15 de cada mês das 15 às 18 horas. O Parque Municipal Tingui foi percorrido em toda sua extensão em busca dos animais e o método utilizado foi a contagem direta 


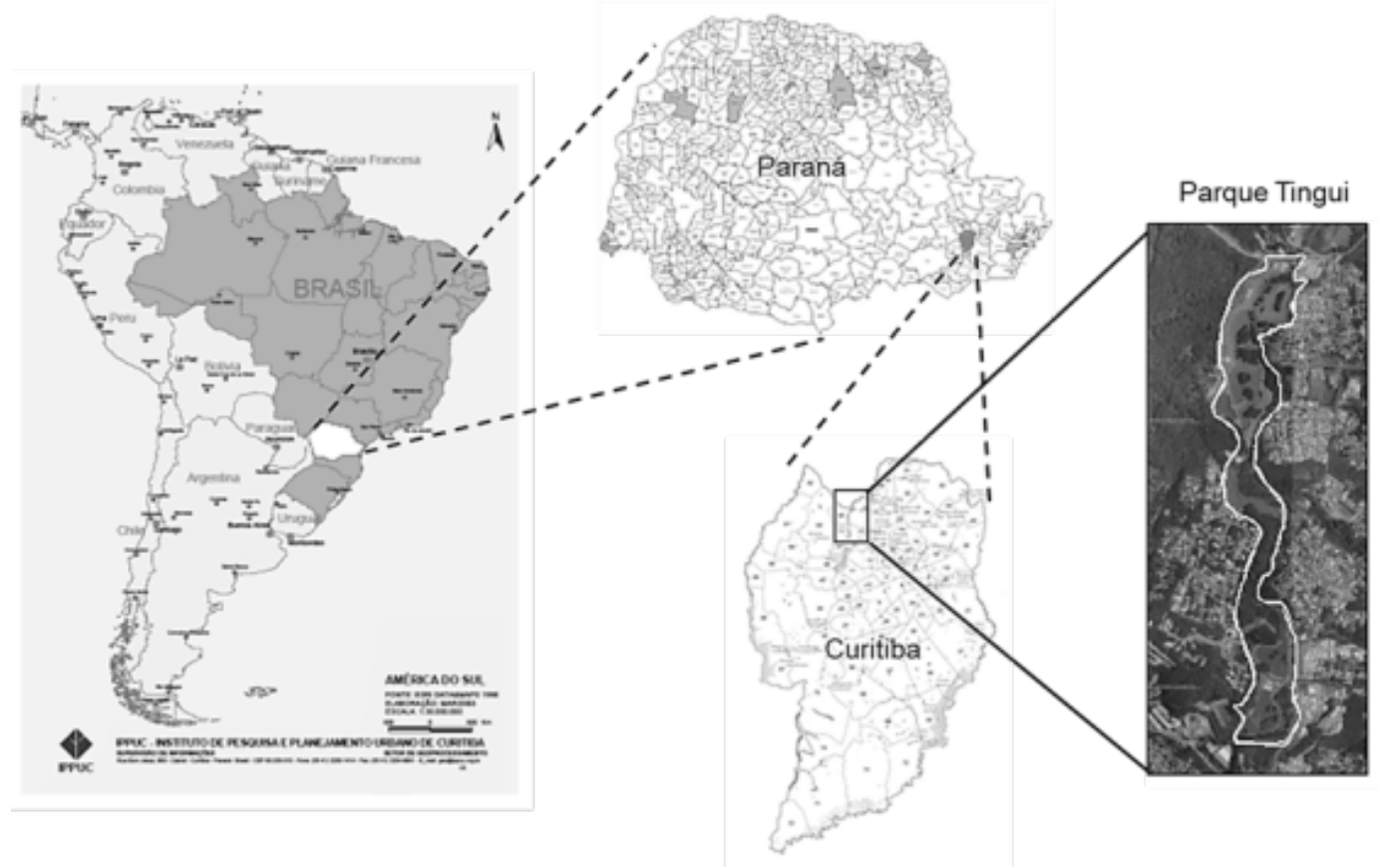

Figura 1. Parque Municipal Tingui, Curitiba-PR

(TOMAZZONI, 2003; MOREIRA \& PIOVEZAN, 2005). Nas observações, foram registrados o número e a composição de animais em cada grupo. Para isso, foi considerado como capivara macho todo adulto com glândula supra nasal localizada na superfície superior do focinho, assim como descrito por vários autores (MONES; OJASTI, 1986; HERRERA, 1992; TOMAZZONI, 2003; COSTA; PAULA, 2006).

Para verificar variações ao longo dos anos a média do número de capivaras, bem como a proporção de adultos e filhotes, foram comparadas pelo teste de Qui-quadrado, a 5\% de significância.

\section{Resultados e Discussão}

A ocorrência das capivaras no Parque Municipal Tingui se deve as características da paisagem que abrange vários lagos, grande extensão de gramado a alguns remanescentes florestais, que compreendem os recursos essenciais a sobrevivência da espécie (ALMEIDA \& BIONDI, 2011).

No que se refere ao tamanho da população, pode-se observar que houve uma queda significativa na média de capivaras de 2008 a 2011 (Tabela 1). Em 2011, o número de indivíduos representou $22 \%$ de 2008 e o número de adultos $25 \%$. Esta redução foi mais intensa para os filhotes que em 2011 chegou a 11,9\% em relação a 2008.
Tabela 1. Média de capivaras no Parque Municipal Tingui, Curitiba-PR

\begin{tabular}{cccc}
\hline \multirow{2}{*}{ Ano } & \multicolumn{3}{c}{ Média \pm Desvio padrão } \\
\cline { 2 - 4 } & Indivíduo & Adulto & Filhote \\
\hline 2008 & $145 \pm 26$ & $108 \pm 16$ & $59 \pm 13$ \\
2009 & $86 \pm 11$ & - & - \\
2010 & $60 \pm 14$ & $52 \pm 11$ & $9 \pm 7$ \\
2011 & $32 \pm 10$ & $27 \pm 7$ & $7 \pm 3$ \\
\hline
\end{tabular}

Apesar da grande queda do número de capivaras nos quatro anos de estudo, a proporção de adultos em 2008 (74\%), 2010 (87\%) e 2011 (84\%) se manteve constante $\left(\chi^{2}=1,13 ; \mathrm{gl}=2 ; \mathrm{P}=0,05\right)$. ALHO et al. (1987a) encontraram proporção semelhante (82\%) em ambiente natural e Tomazzoni (2003) $(82,5 \%) \mathrm{em}$ ambiente sob influência de agricultura e pecuária. Este resultado indica que a proporção de capivaras adultas é equivalente tanto em áreas verdes urbanas como em ambiente natural, de agricultura e de pecuária.

A proporção de filhotes entre 2008 (41\%) e $2010(15 \%)$ diminuiu ( $\chi 2=12,07 ; \mathrm{gl}=1 ; \mathrm{P}=0,05)$ e entre 2010 e $2011(22 \%)$ se manteve constante $(\chi 2=1,32$; $\mathrm{gl}=1 ; \mathrm{P}=0,05)$. A redução entre 2008 e 2010 pode estar relacionada a um aumento de mortalidade por predação ou doenças, visto que é a faixa mais vulnerável (SCHALLER \& CRAWSHAW, 1981; MOREIRA \& MACDONALD, 1997). Em relatório fornecido pela Prefeitura de Curitiba (SILVA, não publicado), foram 


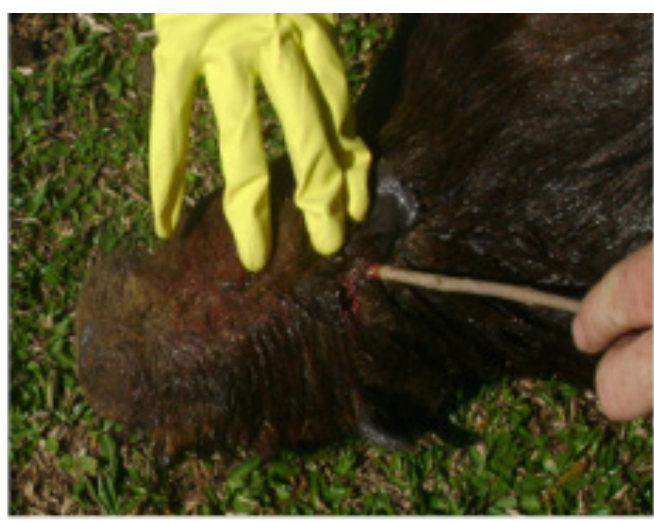

(a)

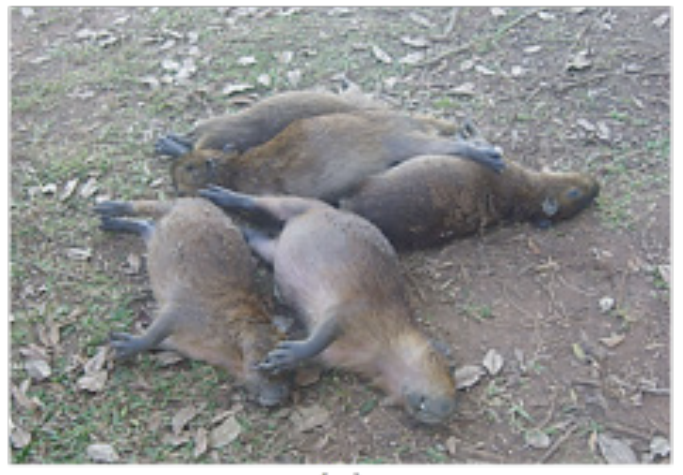

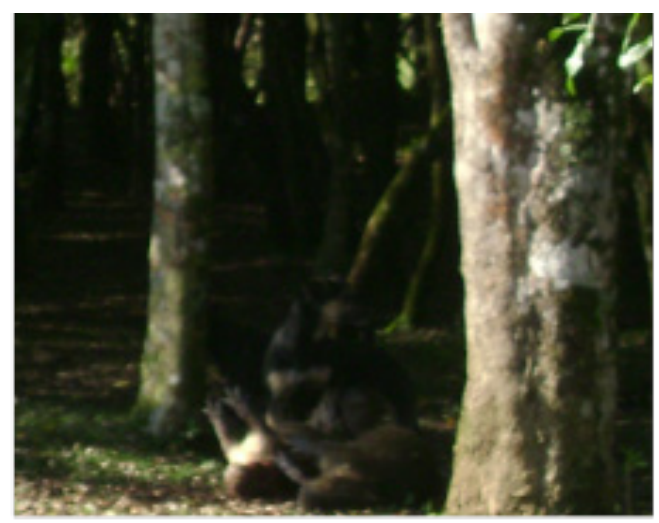

(b)

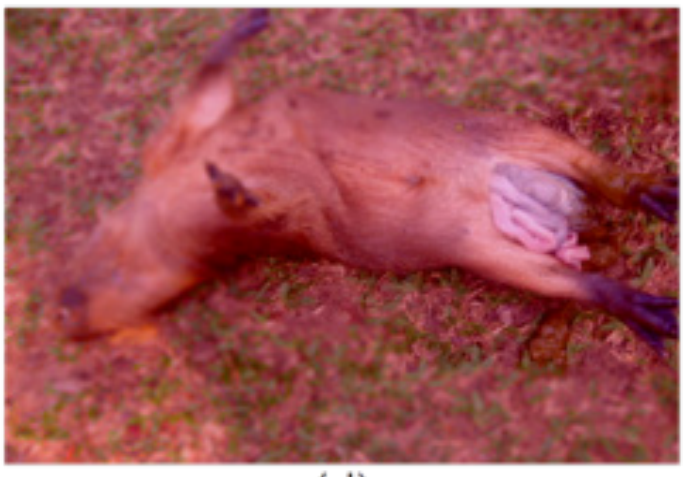

Figura 2. Filhotes de capivara mortos no Parque Municipal Tingui: (a) com perfuração de arma de fogo; (b, c, d) predados por cachorro

Fonte: SILVA, P. W. (2009)

registrados 22 animais mortos em 2009. Destes, 15 eram filhotes com marcas de mordedura de cães e perfuração por arma de fogo (Figura 2). Apesar de serem dados insuficientes para análise, porque não foi realizada uma coleta sistemática, estes registros indicam que a caça e a predação por cachorros podem ser alguns dos motivos que levaram à acentuada redução do número de filhotes entre 2008 e 2010.

Várias doenças afetam este roedor de grande porte, entre elas, as endoparasitoses que causam problemas gastrointestinais e hepáticos que podem levar o animal a óbito (SILVA et al., 2007; BELLATO et al., 2009; OLIVEIRA, 2011). Segundo Truppel (2009), $94 \%$ da população de capivaras da área de estudo apresentou alguma parasitose em 2007, porém não foram observados sinais clínicos de infecção. Apesar desta constatação, os animais podem ter morrido em outros momentos nos quais as condições ambientais desfavoráveis possam ter afetado a resistência imunológica dos animais, refletindo na diminuição do número de capivaras nos anos posteriores. De acordo com Nogueira e Cruz (2007), as parasitoses são mais graves quanto maior a população, isso reforça a hipótese da redução da população entre 2008 e 2010 ter sido causada por doenças, já que em 2008 a população era bem maior que nos três anos seguintes.

Quanto à densidade populacional, ocorreu uma queda gradativa ao longo dos anos, sendo a densidade bruta de 2011 aproximadamente cinco vezes menor que a de 2008 (Figura 3).

$\mathrm{Na}$ área de estudo, a densidade variou de 0,8 a 3,8 capivaras por hectare. Vários autores (ARAÚJO et al., 2007; PEREIRA\& ESTON, 2007; FERRAZ et al., 2001) relataram uma variação de 1,3 a 7 ind/ha em ambientes antropizados. Já em áreas mais naturais as densidades observadas foram de 0,1 a $0,3 \mathrm{ind} / \mathrm{ha}$ (SCHALLER \& CRAWSHAW, 1981; ALHO et al., 1987a; TOMAZZONI, 2003). Neste sentido, a densidade encontrada na área de estudo se mostrou mais semelhante à de ambientes sob influência humana. Isso faz muito sentido já que, de maneira geral, a população de capivaras é mais densa em ambiente modificado devido à maior oferta de recursos e à redução de caça por predadores naturais (FERRAZ et al., 2003; FERRAZ et al., 2007; KRAUER, 2009).

A tendência decrescente da densidade (Figura 3) pode indicar que a capacidade suporte da área do parque possa ter sido atingida e a disponibilidade de recursos (alimento, água e abrigo) encontra-se limitada. Como a capivara ajusta o tamanho do grupo conforme 


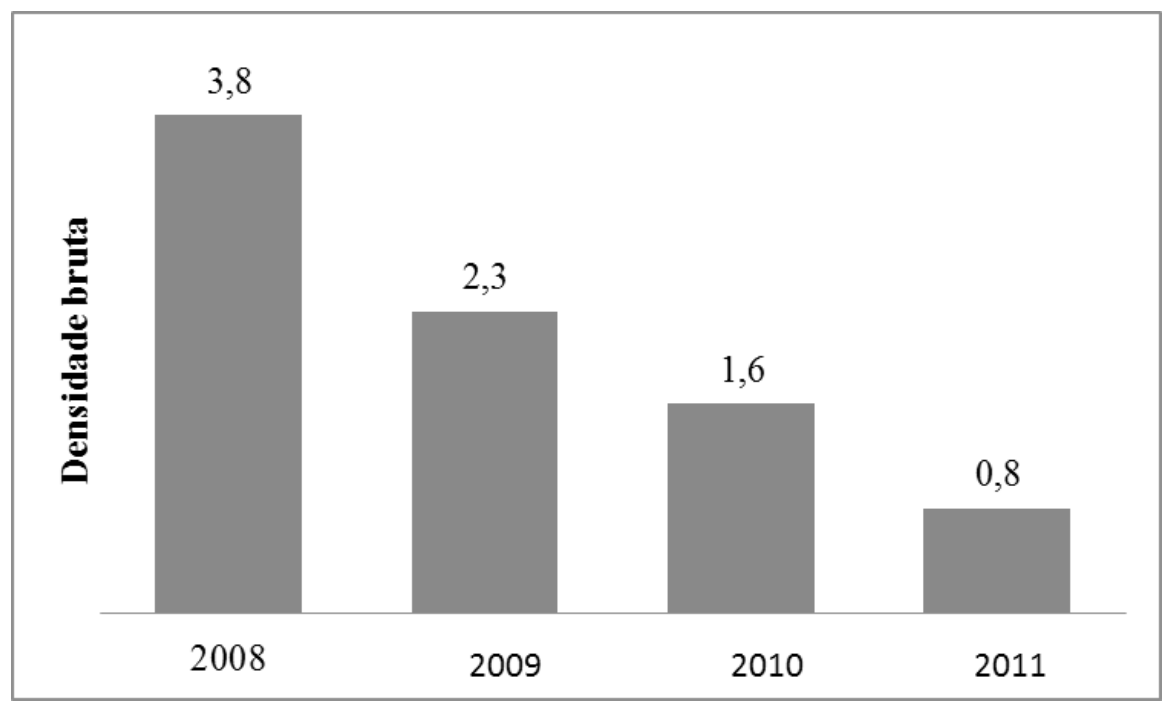

Figura 3. Variação da densidade da população de capivaras do Parque Municipal Tingui, com base no número de indivíduos.

as oscilações de recursos naturais (MOREIRA \& PIOVEZAN, 2005; SOUZA et al, 2007), provavelmente este seja um dos motivos do declínio da população estudada.

De acordo com Odum (2007) e Moreira e Piovezan (2005), a capacidade suporte é o limite máximo sustentável e quando a população atinge este limite, o número de indivíduos se mantém constante, ou seja, não há crescimento populacional. Porém, nesta fase a competição por recursos é grande e leva a população a ultrapassar essa capacidade suporte e a quebrar a sustentabilidade do sistema. Ao ultrapassar este limite o número de indivíduos da população precisa ser reduzido para que o equilíbrio possa ser retomado.
Neste sentido, segundo Pianka (2011), espera-se que ocorra uma elevação da taxa de mortalidade ou uma redução da taxa de natalidade.

Apesar da falta de dados que confirmem a causa da mortalidade e a natalidade, a redução significativa do número de filhotes entre 2008 e 2010 pode ser um indicador de controle populacional. Vários são os comportamentos expressos por um grupo quando há redução de recurso, VARGAS et al. (2007) registrou expulsão de machos em período reprodutivo como medida de diminuição do número de indivíduos e de controle de natalidade em função da escassez de alimento.

O repertório comportamental dos animais exterioriza as influências que o ambiente exerce

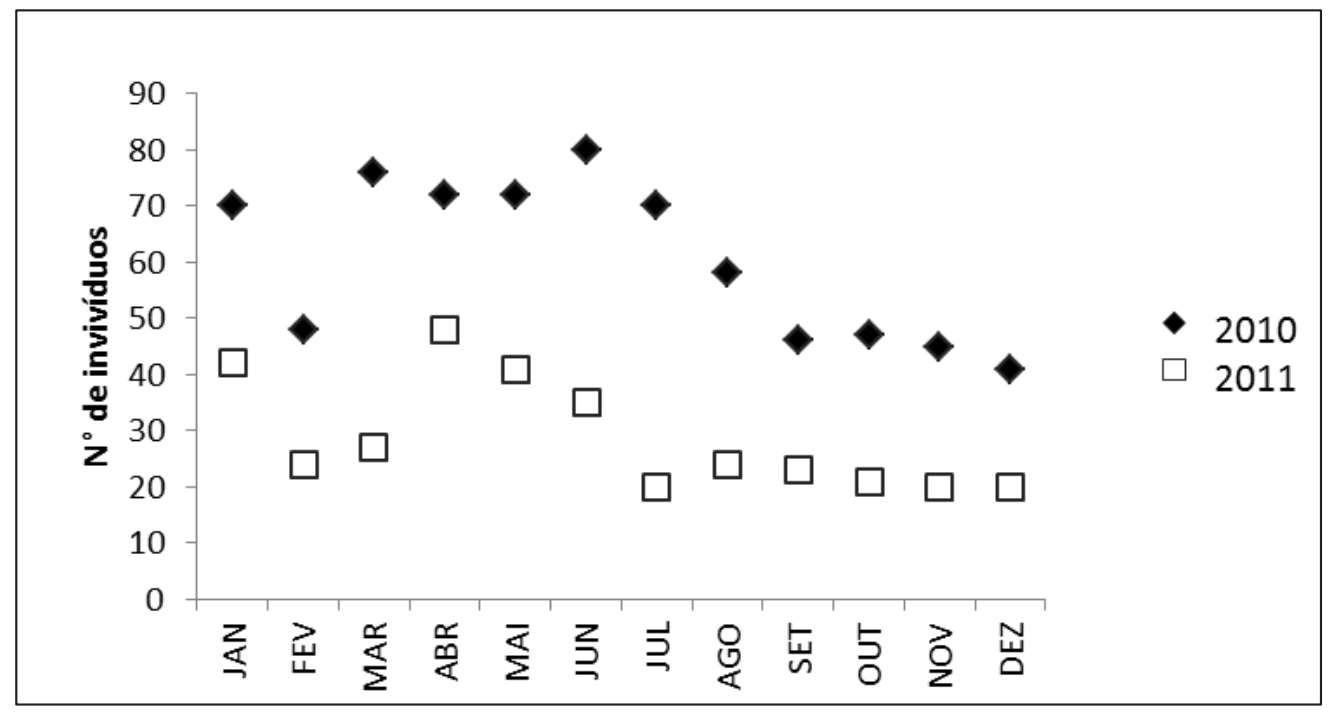

Figura 4. Variação da população de capivaras no Parque Municipal Tingui, PR 
sobre a população. Neste contexto o comportamento territorialista observado por Almeida et al. (2012) evidencia a competição por recursos entre as capivaras do Parque Tingui e reforça a ideia de controle populacional em função do desequilíbrio na oferta de recursos da área.

Outra possível causa da redução de capivaras poderia ser a introdução ou surgimento de espécie competidora (CORRADINI, 2003). Contudo, durante o monitoramento dos animais isso não foi observado, sendo a capivara a única espécie de médio/grande porte herbívora da região.

O aumento do número de capivaras gera competição pelo recurso alimentar e, nestes casos, a espécie tende a otimizar a procura e conversão do alimento e passa a selecionar plantas mais energéticas (ÀLVARES, 2002; ARTEGA \& JORGENSON, 2007). Quanto menor a abundância de alimento, maior a área forrageada e maior a gama de itens alimentares ingeridos para otimizar a razão custo benefício (ODUM, 2007). Se maior é a área forrageada talvez a redução do número de capivaras no parque também se deva a dispersão para áreas vizinhas. Almeida et al. (2013) registraram a presença de capivaras na margem do rio Barigui em trechos que conectam o Parque Municipal Tingui a outros dois parques de características semelhantes.

A dispersão parece acontecer não apenas por recurso alimentar, mas também por abrigo. Em período de enchente (29/janeiro/2011) foi verificada a presença de um grupo de aproximadamente 15 capivaras em propriedade particular próxima a área de estudo, á montante do Rio Barigui (informação fornecida por funcionário da Prefeitura de Curitiba, 2011). Nesta mesma época, foi registrado um número bem menor de capivaras na área (de 42 indivíduos em janeiro para 24 em fevereiro/2011). Isso sugere que os animais tenham saído para se abrigar em propriedades adjacentes, pois segundo Krauer (2009) a dispersão é facilitada pela existência da rede hídrica, que conecta os habitats ainda disponíveis.

Com relação à população de capivaras em 2010 e 2011, a distribuição de indivíduos (Figura 4) indica queda do número de capivaras do mês de janeiro para fevereiro, seguido de um aumento de fevereiro para março. Em 2010, ocorreram dois aumentos de população, março (76 capivaras) e junho (80). Já em 2011 este aumento foi observado apenas em abril (48 indivíduos). Entre os meses de março e julho de 2010 não ocorreu variação $\left(\chi^{2}=0,85 ; \mathrm{gl}=4 ; \mathrm{P}=0,05\right)$, assim como entre agosto e dezembro $\left(\chi^{2}=3,42 ; \mathrm{gl}=4 ; \mathrm{P}=0,05\right)$. Porém, em 2011 esta constância foi entre abril e junho $\left(\chi^{2}=2,05 ; \mathrm{gl}=2 ; \mathrm{P}=0,05\right)$ e de julho a dezembro $\left(\chi^{2}=0,72\right.$; $\mathrm{gl}=5 ; \mathrm{P}=0,05)$.
Durante a pesquisa, ocorreram vários eventos de inundação no Parque Municipal Tingui, porém apenas três foram registrados (dez/2010, jan/2011, jun/2011), sendo o mais severo o que ocorreu em janeiro de 2011. Nesta inundação, grande parte da área de gramado, onde os animais passam a maior parte do dia descansando e forrageando, ficou coberta por uma espessa camada de lama trazida pelo extravasamento do leito do Rio Barigui. Esta lama permaneceu sobre o gamado por aproximadamente 20 dias, período em que as capivaras ficaram impossibilitadas de utilizar os recursos da região. Este acontecimento pode ter induzido os animais a dispersarem em busca de abrigo e alimento podendo ser uma das causas da redução do número de indivíduos de janeiro para fevereiro de 2011. O mesmo pode ter se repetido em julho de 2011.

Ao analisar a variação sazonal da população em 2010 e 2011, pode-se observar que a média máxima de capivaras foi registrada no outono (Tabela 2), um dos períodos de menor precipitação em Curitiba segundo dados do SIMEPAR. Observações semelhantes de maior população durante período de seca foram registradas por Alho et al. (1987a), durante o outono e inverno no Pantanal, e por Tomazzoni (2003), no inverno em uma reserva biológica do Rio Grande do Sul.

Tabela 2. Média e desvio padrão (DP) de capivaras por estação do ano de janeiro de 2010 a dezembro de 2011, Parque Municipal Tingui, Curitiba-PR

\begin{tabular}{ccc}
\hline Ano & Estação & Média \pm DP \\
\hline \multirow{3}{*}{2010} & Verão & $65 \pm 15$ \\
& Outono & $75 \pm 5$ \\
& Inverno & $58 \pm 12$ \\
& Primavera & $44 \pm 3$ \\
\hline \multirow{2}{*}{2011} & Verão & $31 \pm 10$ \\
& Outono & $41 \pm 7$ \\
& Inverno & $22 \pm 2$ \\
& Primavera & $22 \pm 1$ \\
\hline
\end{tabular}

Além do fator clima, outras causas podem influenciar o tamanho da população como, as condições do ambiente e a pressão de predação (ALDANADOMÍNGUEZ et al., 2007). As capivaras deste estudo se encontram em ambiente urbano onde além da área ser restrita, estão expostas a uma maior carga de estresse decorrente do convívio com o homem e suas tecnologias (estradas, carros, etc.). O estresse contínuo pode resultar em alterações metabólicas que interferem na sobrevivência da espécie (BOERE, 2001; BARBOSA \& MOTA, 2004; HOSEY, 2005; DAVID et al., 2005; WELLS, 2005, CAMPELETTO, 2009).

Assim como em ambientes naturais, as capiva- 
ras da área de estudo estão organizadas em grupos ou solitárias. Alguns grupos registrados eram formados apenas por machos, enquanto outros eram mistos, ou seja, apresentaram machos, fêmeas e filhotes. Nas proximidades dos grupos mistos (machos, fêmeas e filhotes), em todos os meses analisados, havia machos solitários que, provavelmente, eram animais satélites que acompanhavam os grupos de origem à distância (ALHO et al., 1987a; ARAÚJO et al., 2007).

Nos grupos mistos, sempre havia pelo menos um macho dominante (cuja glândula supranasal era bem desenvolvida) (Figura 5), um macho subordinado (cuja glândula não era tão evidente), várias fêmeas e filhotes. Nos grupos formados apenas por machos, muitas vezes, todos os integrantes exibiam glândulas nasais bem desenvolvidas. Os grupos eram compostos por dois ou mais animais, em novembro de 2010 e março de 2011 foram registrados grupos de casais, alguns sem filhotes, outros com filhotes e casais com fêmea prenhe. Nos outros meses não foram observados casais.

De maneira geral, os machos solitários estão sempre presentes (MONES \& OJASTI, 1986). Em 2010, no Parque Municipal Tingui, eles representaram 2,8\% da população e em 2011 3,3\%, porcentagens bem menores que a registrada no Pantanal $(7,5 \%)$ por Schaller e Crawshaw (1981).
Entre janeiro de 2010 e dezembro de 2011, a população apresentou uma variação no número e composição de grupos. O número de grupos oscilou entre 2 e 7 e o número de animais em cada grupo variou de dois (macho e fêmea) a 40 (3 machos, 16 fêmea e 21 filhotes) (Figura 6). A proporção entre machos e fêmeas variou de 1:1 a 1:5. No Pantanal, Alho e Rondon (1987) encontraram uma proporção de 1:3 e nas planícies da Venezuela, esta foi de 1:2 (HERRERA \& MACDONALD, 1987). Pinheiro (2007) em sua compilação de índices zootécnicos e biológicos da capivara em diversos sistemas de criação chegou a uma proporção de 1:5 a 1:10. Apesar dos animais do Parque Municipal Tingui se encontrarem em área urbana, pode-se observar que a proporção entre machos e fêmeas é bastante semelhante à encontrada em ambientes naturais.

Embora o parque apresente vários lagos ao longo de toda sua extensão, a população de capivaras se concentra mais ao norte da área (Figura 7A), com exceção de um grupo que foi registrado no extremo sul do Parque Municipal Tingui utilizando fragmento florestal adjacente (Figura 7B). De acordo com Almeida e Daniela (2011) essa concentração de animais está relacionada à proporção de lago e vegetação nesta região.

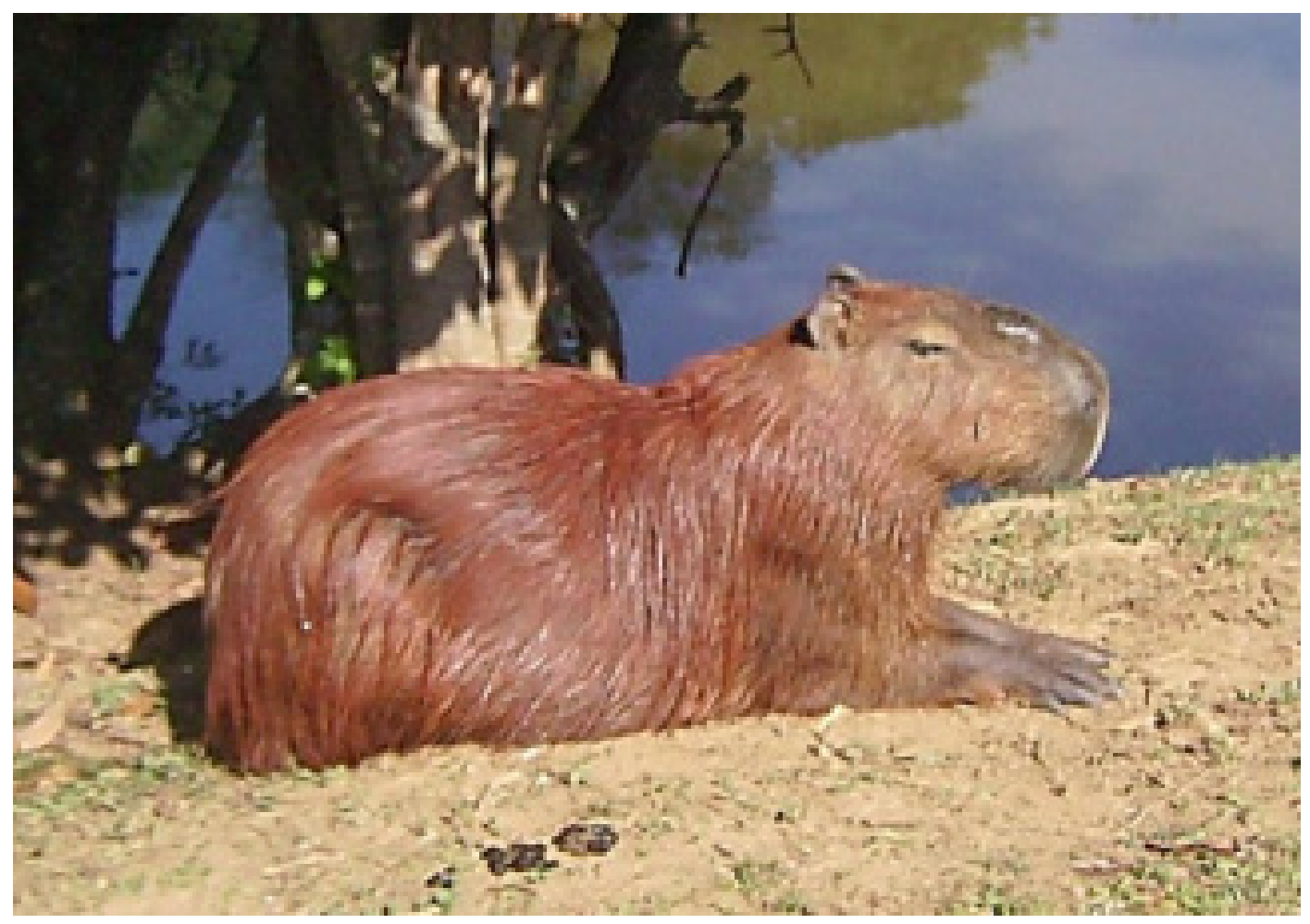

Figura 5. Macho dominante observado no Parque Municipal Tingui, Curitiba-PR 


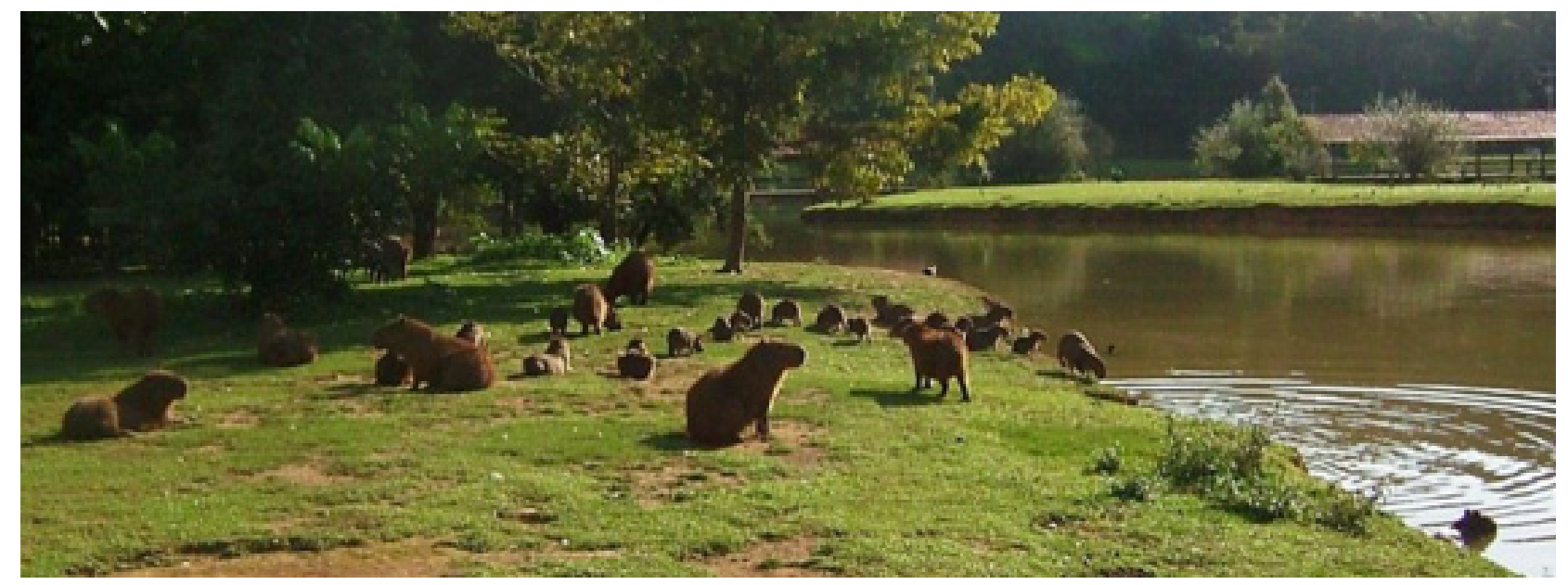

Figura 6. Grupo misto registrado em abril de 2010 no Parque Municipal Tingui
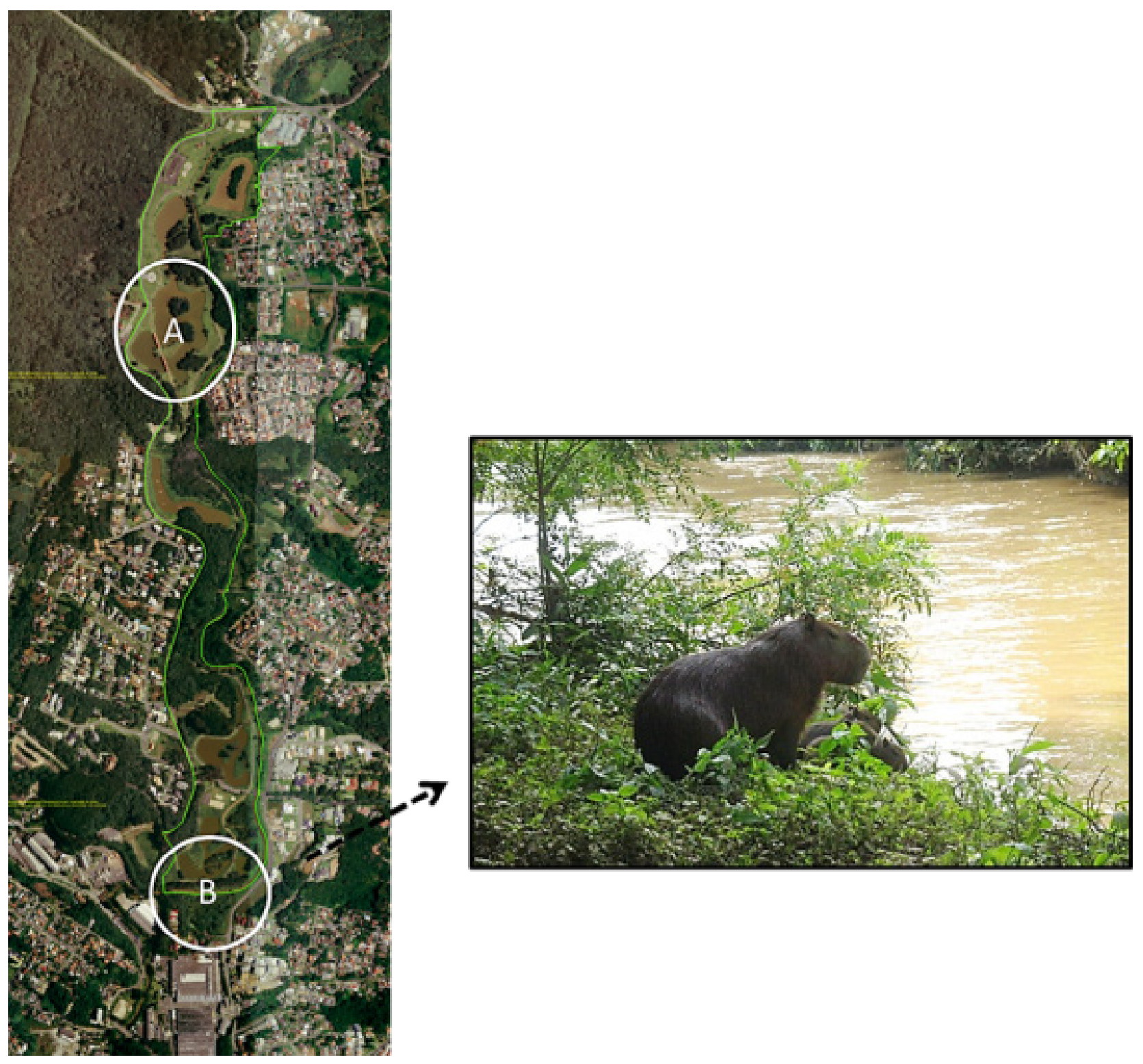

Figura 7. Distribuição da população na área do Parque Municipal Tingui: (A) região norte; (B) região sul com detalhe para grupo registrado em abril de 2010 


\section{Conclusão}

A partir dos resultados obtidos, pode-se concluir que apesar das capivaras se encontrarem em ambiente urbano, a estrutura e composição da população são muito semelhantes a dos animais que vivem em áreas mais preservadas. Isso evidencia o elevado grau de tolerância que a espécie possui para viver em ambientes desta natureza, além de reforçar a importância das áreas verdes urbanas nas grandes cidades na conservação de espécies silvestres.

\section{Referências}

ALDANA-DOMÍNGUEZ, J.; VIEIRA-MUÑOZ, M.I.; ÁNGEL-ESCOBAR, D.C. Estudios sobre la ecologia del chigüiro (Hydrochoerus hydrochaeris), enfocados a su manejo y uso sostenible en Colombia. Instituto Alexander von Humboldt. Colômbia: Bogotá D.C., 2007, 188 p.

ALHO, C.J.R.; CAMPOS, Z.M.S.; GONÇALVES, H.C. Ecologia de capivara (Hydrochaeris hydrocharis, Rodentia) do Pantanal: habitats, densidade e tamanho de grupo. Revista Brasileira de Biologia, v.47, n.1/2, p.99-110, 1987a.

ALHO, C.J.R.; CAMPOS, Z.M.S.; GONÇALVES, H.C. Ecologia de capivara (Hydrochaeris hydrocharis, Rodentia) do Pantanal: atividade, sazonalidade, uso do espaço e manejo. Revista Brasileira de Biologia, v.47, n.1/2, p.99-110, 1987b.

ALHO, C.J.R.; RONDON, N.L. Habitats, population densities, and social structure of capybaras (Hydrochaeris hydrochaerls, Rodentia) In the Pantanal, Brazil. Revista Brasileira de Zoologia, v.4, n.2, p.139-149, 1987.

ALMEIDA, A.R.; BIONDI, D. Estudo da paisagem e a ocorrência de capivaras no Parque Municipal Tingui - Curitiba-PR. Revista de Ciências Agrárias, v.54, n.3, p.280-289, 2011.

ALMEIDA, A.R.; BIONDI, D.; MONTEIROFILHO, E.A.L. Comportamento de capivaras em área verde urbana no município de Curitiba, PR. Revista Biociências, Taubaté, v. 18, n.1, p. 24-31, 2012.

ALMEIDA, A.M.R.; ÁRZUA, M.; TRINDADE, P.W.S.; SILVA JUNIOR, A. Capivaras (Hydrochoerus hydrochaeris, Linnaeus, 1766)
(Mammalia: Rodentia) em áreas verdes do município de Curitiba (PR). Estudos de Biologia, v.35, n.84, p.9-16, 2013.

ALMEIDA, A.R.; LEAL, L.; BIONDI, D.; MARTINI, A.; LIMA NETO, E.M. Caracterização microclimática do Parque Municipal Tingui, Curitiba-PR e a ocorrência de capivaras (Hydrochoerus hydrochaeris, Lunnaeus, 1766). REVSBAU, v.8, n.2, p.46-57, 2013.

ÁLVAREZ, M.R. Manejo sustentable del carpincho (Hydrochoerus hydrochaeris, LINNAEUS 1766) en Argentina: um aporte al conocimiento de la biología de la especie desde la cría em cautiverio. 2002. 210 f. Tese (Doutorado), Universidade de Buenos Aires, 2002.

ARAÚJO, R.A.; ALMEIDA, A.J.; TORQUETTI, C.G.; FICHE, H.; ALAMONI, S.A. Monitoramento de um grupo de capivaras Hydrochaerus hydrochaeris (Mammalia: Rodentia) em um parque urbano na região da Pampulha, Belo Horizonte, Brasil. In: CONGRESSO DE ECOLOGIA DO BRASIL, 8, 2007, Caxambu. Anais...Caxambu: CEB, 2007.

ARTEAGA, M.C.; JORGENSON, J.C. Habitos de desplazamiento y dieta del capibara (Hydrochoerus hydrochaeris) em la Amazonia Colombiana. Mastozoología Neotropical, v.14, n.1, p.11-17, 2007.

BARBOSA, M.N.; MOTA, M.T.S. Influência da rotina de manejo na interação social entre pares heterosexuais do sagüi, Callithrix jacchus (Linnaeus, 1758). Revista Brasileira de Zoociências, v. 6, n.1, p.29-43, 2004.

BELLATO, V.; SOUZA, A.P.; SARTOR, A.A.; VEIGA, L.P.H.N.; CENTENARO, F. Ocorrência de Fasciola hepatica na população de capivaras (Hydrochaeris hydrochaeris) e em bovinos (Bostaurus) no município de Timbó, SC. Revista de Ciências Agroveterinárias , v.8, n.1, p. 66-70, 2009.

BOERE, V. Environmental enrichment for neotropical primates in captivity. Ciência Rural, v.31, n.3, p.543-551, 2001.

CAMPELETTO, A.J. Efeitos do clima tropical sobre os animais de interesse zootécnico. Bioclimatologia animal online. Disponível em 
$<$ http://www.bioclima.info/capivara.php > Acesso em: 30 Ago 2012.

CORRADINI, A.P. Avaliação de índices zootécnicos e metodologia para aplicação em manejo sustentável de capivaras (Hydrochaeris hydrochaeris), em Pirassununga/SP. 2003. 116f. Dissertação (Mestrado em Zootecnia), Faculdade de Zootecnia e Engenharia de Alimentos, Universidade de São Paulo. 2003.

COSTA, D.S.; PAULA, T.A.R. Testosterone level, nasal gland volume and Leydig cell morphometry in capybaras (Hydrochoerus hydrochaeris). Arquivos Brasileiros de Medicina Veterinária e Zootecnia, v.58, n.6, p.1086-1091, 2006.

DAVID, N.; SCHAFFNER, C.M.; SMITH, T.E. Evidence that zoo visitors influence HPA activity in spider monkey (Ateles geoffroyii rufiventris). Applied Animal Behaviour Science, v.90, p. 131141, 2005.

DEUTSCH, L.A.; PUGLIA, R.R. Os animais silvestres: proteção, doenças e manejo. São Paulo: Globo, 1990, 191 p.

ECOTÉCNICA. Plano de manejo do Parque Tingui. Curitiba. 2009. Disponível em: <http:// www.curitiba.pr.gov.br/conteudo/plano-demanejo-smma/322> Acesso em 18 Set 2013.

EMMONS, L.H.; FEER, F. Neotropical rainforest mammals: a field guide. The University of Chicago Press, Chicago and London, 1997, 281 p.

FERRAZ, K.P.M.B.; SANTOS-FILHO, R.M.F.; PIFFER, T.R.O.; VERDADE, L.M. Biologia e manejo da capivara: do controle de danos ao máximo rendimento sustentável. In: A Produção Animal na Visão dos Brasileiros, Piracicaba: FEALQ, 2001, v.1, p.580-588.

FERRAZ, K.M.P.M.B.; LECHEVALIER, M.; COUTO, H.T.Z.; VERDADE, L.M. Damage caused by capybaras in a corn field. Scientia Agricola, v.60, n.1, p.191-194, 2003.

FERRAZ, K.M.P.M.B.; FERRAZ, S.F.B.; MOREIRA, J.R.; COUTO, H.T.Z.; VERDADE, L.M. Capybara (Hydrochoerus hydrochaeris) distribution in agroecosystems: a crossscale habitat analysis. Journal of Biogeography, v.34, p.223-230, 2007.
FERRAZ, K.M.P.M.B.; PETERSON, A.T.; SCACHETTI-PEREIRA; VETTORAZZI, C.A.; VERDADE,L.M. Distribution of capybara in an agroecosystem Brazil, based on ecological niche modeling. Journal of Mammalogy, v.90, n.1, p.189194, 2009 .

HERRERA, E.A.; MACDONALD, D.W. Group stability and structure of a capybara population. Symposia of the Zoological Society of London, n.58, p.115-130, 1987.

HERRERA, E.A. Size of tests and a scent glands in capybaras Hydrochaeris hydrochaeris (Rodentia: Caviomorpha). Journal of Mammalogy, v.3, n.4, p.: 871-875, 1992.

HOSEY, G.R. How does the zoo environmental affect the behaviour of captive primates? Applied Animal Behaviour Science, v. 90, p. 107-129, 2005.

IPPUC - Instituto de Pesquisa e Planejamento Urbano de Curitiba. Curitiba em dados: áreas verdes. 2011. Disponível em: <http://www.ippuc.org.br/ Bancodedados/Curitibaemdados/Curitiba_em dados_Pesquisa.htm>. Acesso em: 20 Ago. 2012

KRAUER, J.M.C. landscape ecology of the capybara (Hydrochoerus hydrochaeris) in the Chaco region of Paraguay. 2009. 128 f. Tese (Doutorado em Filosofia), Divisão de Biologia, Universidade do Kansas, 2009.

MACDONALD, D.W. Dwindling resources and the social behaviour of capybara, Hydrochoerus hydrochaeris) (Mammalia). Journal of Zoological of London, v.194, p. 371-391, 1981.

MONES, A.; OJASTI, J. Hydrochoerus hydrochaeris. Mammalian Species, n.264, p.1-7, 1986.

MOREIRA, J.R.; MACDONALD, D.W. Técnicas de manejo de capivaras e outros grandes roedores na Amazônia. In: VALLADARES-PADUA, C.; BODMER, R.E; CULLER, L. Manejo e conservação da vida Silvestre no Brasil. Brasília: Sociedade Civil Mamirauá, 1997, p.186-213.

MOREIRA, J.R.; PIOVEZAN, U. Conceitos de manejo de fauna, manejo de população problema e o exemplo da capivara. Brasília: Embrapa Recursos Genéticos e Biotecnologia, 2005, 23 p. 
NOGUEIRA, M. F.; CRUZ, T. F. Doenças da capivara. Corumbá: Embrapa Pantanal, 2007. 74p.

ODUM, E.P. Fundamentos de Ecologia. São Paulo: Thomson Learning, 2007, 612 p.

OLIVEIRA, J.A.; SILVEIRA, G.; ROCHA, V.J.; SILVA, C.E.F. Ordem Rodentia. In: REIS, N.R.; PERACCHI, A.L.; FANDIÑO-MARIÑO, H; ROCHA, V.J. Mamíferos da Fazenda Monte Alegre, Paraná. Londrina: EDUEL. 2005, p.161-191.

OLIVEIRA, U.C. Eimeria spp. de coelho e galinha domésticos: desenvolvimento de ensaios moleculares e caracterização filogenética. 2011. 44 f. Tese (Doutorado em Ciências), Universidade de São Paulo, São Paulo. 2011.

OLIVEIRA, J.A.; BONVICINO, G.R. Ordem Rodentia. In: REIS, N. R.; PERACCHI, A. L.; PEDRO, W. A.; LIMA, I. P. Mamíferos do Brasil. Londrina: Nélio R. dos Reis, 2011, p.358-433.

PEREIRA, H.F.A.; ESTON, M.R. Biologia e manejo de capivaras (Hydrochoerus hydrochaeris) no parque estadual Alberto Löfgren, São Paulo, Brasil. Rev. Inst. Flor., v.19, n.1, p.55-64, 2007.

PIANKA, E.R. Evolutionary Ecology. United States of America: eBook. 2011. 431p.

PINHEIRO, M.S. Criação de capivara em sistema Intensivo. Pelotas: Embrapa, 2007. Disponível em <http://www.cpact.embrapa.br/publicacoes/ download/folder/capivara.pdf $>$. Acesso em: 30 Ago 2012.

SCHALLER, G.S.; CRAWSHAW,P.G. Social organization in a capybara population. Saugetierkundliche Mitteilungen, v.29, p.3-16, 1981.

SILVA, M.K.S.; SILVA, A.S.; OLIVEIRA, C.B.; SOARES, J.F.; MONTEIRO, S.G. Ocorrência de Eimeria

ichiloensisem em capivaras (Hydrochaeris hydrochaeris) de criatório. Arquivo de Ciências Veterinárias e Zoologia da UNIPAR, v.10, n.2, p. 129-131, 2007.

SOUZA, D.C.; OLIVEIRA, M.A.; NEIVERTH, A. NASCIMENTO JÚNIOR,A.F. Produção de painéis montáveis de ímã como Recurso para o ensino de ecologia a partir do comportamento da capivara (Hydrochoerus hydrochoeris). Fórum Ambiental da Alta Paulista, v.3, 2007, 10p.

TOMAZZONI, A.C. Ecologia da capivara (Hydrochoerus hydrochaeris, Linnaeus, 1766) ( Mammalia, Rodentia) na Reserva Biológica do Lami, sul do Brasil. 2003. 81 f. Dissertação (Mestrado em Ecologia), Universidade Federal do rio Grande do Sul, Porto Alegre, 2003.

TRUPPEL, J.H. Avaliação do parasitismo em capivaras (Hydrochaeris hydrochaeris) e sua atuação como hospedeiro intermediário de Neospora caninume Toxoplasma gondii. 194 f. Mestrado (Ciências Biológicas e da Saúde), Universidade Federal do Paraná, Curitiba, 2009.

VARGAS, F.C; VARGAS, S.C.; MORO, M.E.G.; SILVA, V.; CARRER, C.R.O. Monitoramento populacional de capivaras (Hydrochaeris hydrochaeris Linnaeus, 1766) em Pirassununga, SP, Ciência Rural, v.37, n.4, p.1104-1108, 2007.

WELLS, D.L.A note on the influence of visitors on the behaviour and welfare of zoo-housed gorillas. Applied Animal Behaviour Science, v. 93, p.13-17, 2005. 